\title{
The Chemical Biology of Hydropersulfides (RSSH): Chemical Stability, Reactivity and
}

Redox Roles

Simran S. Saund ${ }^{1}$, Victor Sosa ${ }^{1}$, Stephanie Henriquez ${ }^{2}$, Q. Nhu N. Nguyen ${ }^{3}$, Christopher L. Bianco $^{1}$, Shuhei Soeda ${ }^{1}$, Robert Millikin ${ }^{1}$, Corey White ${ }^{1}$, Henry Le ${ }^{1}$, Katsuhiko Ono ${ }^{1}$, Dean J. Tantillo $^{3}$, Yoshito Kumagai ${ }^{4}$, Takaaki Akaike ${ }^{5}$, Joseph Lin ${ }^{2 *}$ and Jon M. Fukuto ${ }^{1 *}$

${ }^{1}$ Department of Chemistry, Sonoma State University, Rohnert Park, CA 94928, ${ }^{2}$ Department of Biology, Sonoma State University, Rohnert Park, CA 94928, ${ }^{3}$ Department of Chemistry, University of California, Davis, One Shield Ave., Davis, CA 95616, ${ }^{4}$ Environmental Biology Section, Faculty of Medicine, University of Tsukuba, Tsukuba, Ibaraki 305-8575, Japan, ${ }^{5}$ Department of Environmental Health Sciences and Molecular Toxicology, Tohoku University Graduate School of Medicine, Sendai 980-8575, Japan

*to whom correspondence can be addressed. Jon M. Fukuto, 7076642187 , fukuto@sonoma.edu or Joseph Lin, 707664 2931, linj@sonoma.edu. 


\section{Graphical abstract}

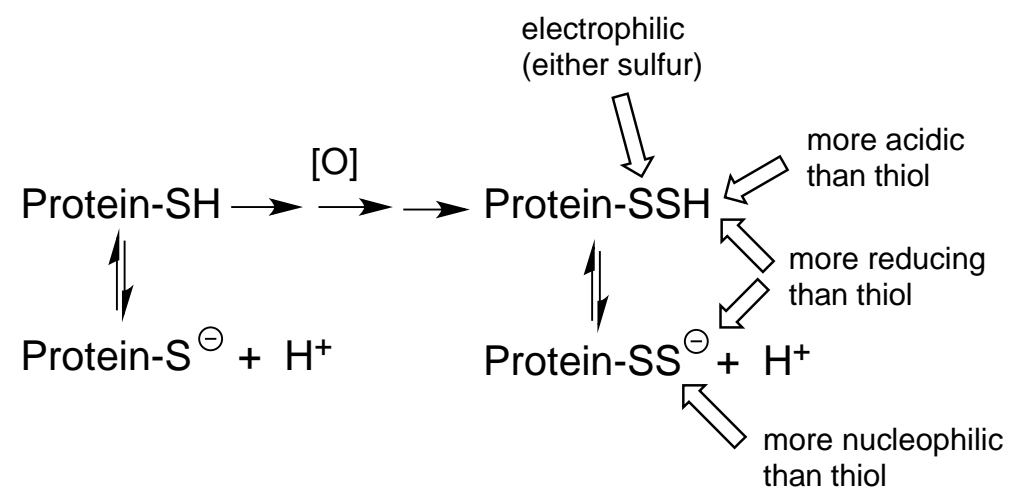


Keywords: Hydropersulfide, thiols, redox regulation, nucleophilicity, electrophilicity.

Abbreviations: tris(2-carboxyethyl)phosphine hydrochloride (TCEP), dithiothreitol (DTT), 2hydroxyethyl disulfide (HE-SS-HE), fluorescein diphosphate (FDP), highest occupied molecular orbital (HOMO), lowest unoccupied molecular orbital (LUMO). 


\section{Abstract}

Recent reports indicate the ubiquitous prevalence of hydropersulfides (RSSH) in mammalian systems. The biological utility of these and related species is currently a matter of significant speculation. The function, lifetime and fate of hydropersulfides will be assuredly based on their chemical properties and reactivity. Thus, to serve as the basis for further mechanistic studies regarding hydropersulfide biology, some of the basic chemical properties/reactivity of hydropersulfides were studied. The nucleophilicity, electrophilicity and redox properties of hydropersulfides were examined under biological conditions. These studies indicate that hydropersulfides can be nucleophilic or electrophilic, depending on the $\mathrm{pH}$ (i.e. the protonation state) and can act as good one- and two-electron reductants. These diverse chemical properties in a single species make hydropersulfides chemically distinct from other, well-known sulfur containing biological species, giving them unique and potentially important biological function.

\section{Introduction}

The small-molecule signaling agents $\cdot \mathrm{NO}, \mathrm{CO}$ and $\mathrm{H}_{2} \mathrm{~S}$ are endogenously generated and presumed to possess important signaling function [for a review, see 1]. In spite of the reported biological importance of $\mathrm{H}_{2} \mathrm{~S}$ [for example, 2], the chemistry by which $\mathrm{H}_{2} \mathrm{~S}$ acts as a biological signaling or effector species is not well understood. Biosynthesis of $\mathrm{H}_{2} \mathrm{~S}$ can involve initial generation of hydropersulfides (RSSH), which can subsequently release $\mathrm{H}_{2} \mathrm{~S}$ under reducing conditions (vide infra). However, recent reports indicate that hydropersulfides are prevalent and may be themselves relevant and ubiquitous biological effector and/or signaling species [for example, 3-10]. (Note: the term "hydropersulfide" as used herein refers to both the protonated RSSH and deprotonated RSS forms unless otherwise indicated). Indeed, it has been proposed that hydropersulfides, and not $\mathrm{H}_{2} \mathrm{~S}$, are major and important effector species and that $\mathrm{H}_{2} \mathrm{~S}$ in many cases may be more of a marker for their presence $[3,11]$. To be sure, hydropersulfides have been reported many years ago as important functionalities in several enzymes and/or proteins [for example, 12-17]. Although the presumed biological utility of hydropersulfides is predicted to be based on their unique chemistry (that appears to be distinct from the biological chemistry of thiols) [18], the chemical details of the actions of biological hydropersulfides have not been substantiated. That is, it appears that hydropersulfides may have important biological 
function and unique chemistry but it is not yet fully understood what aspects of this chemistry may be important to their biological utility. In order to understand the chemical biology of hydropersulfides, it is important to delineate all of the possible reactions of this functionality under biological conditions. Thus, examination of some of the fundamentally important chemical properties of hydropersulfides was undertaken in an attempt to begin to understand their fate, reaction with relevant biological targets, general stability and potential biological utility.

\section{Experimental Methods}

Materials/Reagents: Glutathione, cystine, cysteine, glutathione-agarose, hydroxylamine-hydrochloride, hydrazine solution, tris(2-carboxyethyl)phosphine hydrochloride (TCEP), hydroxylamine hydrochloride $\left(\mathrm{NH}_{2} \mathrm{OH} \cdot \mathrm{HCl}\right)$, anhydrous hydrazine $\left(\mathrm{H}_{2} \mathrm{NNH}_{2}\right)$, sodium azide $\left(\mathrm{NaN}_{3}\right)$ and dithiothreitol (DTT) were all obtained from Sigma-Aldrich (St. Louis, MO). Sodium hydrogen sulfide (NaHS) was purchased from Strem Chemicals, Inc. (Newburyport, MA). Sodium sulfide $\left(\mathrm{Na}_{2} \mathrm{~S}\right)$ and sodium tetrasulfide $\left(\mathrm{Na}_{2} \mathrm{~S}_{4}\right)$ was purchased from Alfa Aesar (Ward Hill, MA). Immobilized TCEP resin was purchased from Thermo Scientific (Waltham, MA), 2-hydroxyethyl disulfide (HE-SS-HE) was purchased from Acros Organics (Fair Lawn, NJ) and fluorescein diphosphate was purchased from AnaSpec (Fremont, CA). All other chemicals and reagents were purchased from commercial suppliers and were of the highest purity available. $\mathrm{Cu}^{\prime}\left(\mathrm{CH}_{3} \mathrm{CN}\right)_{4} \mathrm{ClO}_{4}$ was synthesized as previously described [19].

Instrumentation: ${ }^{1} \mathrm{H}-\mathrm{NMR}$ spectra were obtained using an Agilent $400 \mathrm{MHz}$ spectrometer. Dissolved oxygen measurements were performed using a YSI 5300 Biological Oxygen Monitor. Absorbance spectra were obtained using a Shimadzu 2501PC spectrophotometer. Biochemical fluorescence assays were performed on a BioTek Synergy HT Multi-Mode Microplate Reader.

\section{Reaction of $\mathrm{CN}^{-}$Anion and $\mathrm{O}_{2}$ with 2-Hydroxyethyl Persulfide (HE-SSH): 2-Hydroxyethyl} disulfide (HE-SS-HE) was diluted into $100 \mathrm{mM}$ glycine buffer, $\mathrm{pH} 10$ or $500 \mathrm{mM}$ phosphate buffer, $\mathrm{pH} 7.2$ to a final concentration of $10 \mathrm{mM}$ in a quartz cuvette. $\mathrm{Na}_{2} \mathrm{~S}$ was then added to a final concentration of $10 \mathrm{mM}$ and immediately sealed from atmosphere. The absorbance at 340 $\mathrm{nm}$ (RSS) was monitored continuously. The reaction was allowed to proceed undisturbed for $1,500 \mathrm{sec}$. in order to establish the equilibrium between disulfide $/ \mathrm{H}_{2} \mathrm{~S}$ and hydropersulfide/thiol (vide infra). After 25 minutes, $\mathrm{NaCN}$ was added to give approx. $1.0 \mathrm{mM}$ and $0.5 \mathrm{mM} \mathrm{CN}^{-}$(note: 
approximately $90 \mathrm{X}$ more $\mathrm{NaCN}$ was added to the cuvette at $\mathrm{pH} 7.2$ compared to $\mathrm{pH} 10$ in order to achieve equal molar $\mathrm{CN}^{-}$levels in the two experiments) or the cuvette solution was sparged with either at stream of $\mathrm{N}_{2}, \mathrm{O}_{2}$ or atmosphere and the cuvette resealed. The loss of absorbance at $340 \mathrm{~nm}$ was then monitored to measure the loss of the persulfide anion from solution.

Examination of the Reactions of Nucleophiles with 2-Hydroxyethyl Persulfide by NMR: HE-SS$\mathrm{HE}$ and $\mathrm{Na}_{2} \mathrm{~S}$ were mixed in a $\mathrm{D}_{2} \mathrm{O} /$ borate buffer ( $500 \mathrm{mM}, \mathrm{pH} 10$ ) solution to a final concentration of $20 \mathrm{mM}$ and placed in an NMR tube. After 25 minutes reaction, $\mathrm{H}_{2} \mathrm{NOH} . \mathrm{HCl}$, $\mathrm{H}_{2} \mathrm{NNH}_{2}, \mathrm{NaN}_{3}$ or $\mathrm{NaCN}$, taken up in the same solvent mixture, was added to the reaction solution to final concentrations of $100 \mathrm{mM}$. ${ }^{1} \mathrm{H}$-NMR Spectra (using water suppression) were then taken after 1 hour.

$\underline{\mathrm{O}}_{2}$-Consumption by Hydropersulfides: Experiments were performed at room temperature in a sealed system using an YSI Model 5300 Biological Oxygen Monitor equipped with a Clark oxygen electrode. The initial buffer solution was set as $100 \%$. Reagents were then added via a syringe with an elongated needle and $\mathrm{O}_{2} \%$ was monitored continuously over time. For Figure 4B stock solutions of $\mathrm{Na}_{2} \mathrm{~S}$, NaHS, GSSG, and GSH were all prepared in $100 \mathrm{mM}$ Tris, pH 7.4. Glutathione persulfide was made by first equilibrating the $\mathrm{O}_{2}$ monitor in $3.0 \mathrm{~mL}$ of $100 \mathrm{mM}$ Tris, pH 7.4 buffer. Next, $100 \mu \mathrm{L}$ of $30 \mathrm{mM}$ GSH was added followed by $100 \mu \mathrm{L}$ of $3.0 \mathrm{mM} \mathrm{Na}_{2} \mathrm{~S}$. Finally, $100 \mu \mathrm{L}$ of $30 \mathrm{mM}$ GSSG was added. $\mathrm{O}_{2} \%$ was monitored for several minutes after the addition of each reagent.

Removal of Polysulfide Impurities from $\mathrm{Na}_{2} \underline{S}_{4}: \mathrm{Na}_{2} \mathrm{~S}_{4}$ in $1 \mathrm{M}$ Tris, $\mathrm{pH} 8.2$ was added to TCEP resin at a final concentration of $8.3 \mathrm{mM} \mathrm{Na}_{2} \mathrm{~S}_{4}$, and $9.1 \mathrm{mM} \mathrm{TCEP}$ resin (calculated) for 15-30 minutes at room temperature. Reactions were mixed every 3-4 min to prevent settling of the resin. After incubation, the resin was removed by centrifugation. The sample was then injected into $100 \mathrm{mM}$ glycine, $\mathrm{pH} 10$ buffer to analyze oxygen consumption to a final concentration of 280 $\mu \mathrm{M} \mathrm{Na}_{2} \mathrm{~S}_{4}$.

Computational Studies: Optimization and frequency calculations for minima and transition state structures (TSSs) were performed in water, modeled with the SMD implicit continuum solvation model [20] using the M06-2X/6-31+G(d,p) method [21] implemented in Gaussian09 [22]. Intrinsic reaction coordinate (IRC) calculations were also performed to confirm which minima are connected to TSSs [23,24]. Minima used to compute proton affinities and electrostatic potential 
maps (isovalue $=0.0004$ and potential range of -0.03 to 0.03 ) were located in both water and the gas phase using both the M06-2X/6-31+G(d,p) and B3LYP/6-31+G(d,p) methods $[25,26$, 27].

Recombinant CSE: Rat CSE was cloned into pGEX-6P-3 via Xhol and Notl followed by transformation into E. coli (T7 Express, New England BioLabs). The cells were cultured in LB containing ampicillin $(100 \mu \mathrm{g} / \mathrm{mL})$ at $37^{\circ} \mathrm{C}$ for $3 \mathrm{~h}$, and induced at $500 \mu \mathrm{M}$ IPTG at $30{ }^{\circ} \mathrm{C}$ for 10 h. Cells were harvested and lysed in $50 \mathrm{mM}$ Tris, pH 7.4, $150 \mathrm{mM} \mathrm{NaCl}$, lysozyme, DNAse I, and a Protease Inhibitor Cocktail (Roche) followed by sonication. Pyridoxal phosphate (50 $\mu \mathrm{M})$ and DTT ( $1 \mathrm{mM})$ was added during the binding $\left(4^{\circ} \mathrm{C}\right.$ for $\left.2 \mathrm{~h}\right)$ of GST-CSE to GlutathioneAgarose (Sigma-Aldrich) and then washed with 50 mM HEPES buffer.

Recombinant CD148: The cytoplasmic domain of human CD148 (R997 to the C-term) was cloned into pTrcHis-A (Invitrogen; Grand Island, NY) to include a 10xHis N-term tag. The plasmid was transformed into Arctic Express bacteria (Agilent; Santa Clara, CA) and induced with IPTG for 18 hours at $10^{\circ} \mathrm{C}$. Cells were lysed in lysis buffer (50 mM HEPES pH 7.2, 300 $\mathrm{mM} \mathrm{NaCl}, 10 \mathrm{mM}$ imidazole, $1 \mathrm{mg} / \mathrm{ml}$ lysozyme, DNAse, and a protease inhibitor cocktail (Roche)) followed by sonication and centrifugation at 8,000 $\mathrm{xg}$. Protein was purified from cell lysates using Talon resin (Clontech; Mountain View, CA), then oxidized with $100 \mu \mathrm{M} \mathrm{H}_{2} \mathrm{O}_{2}$ for 15 min., next treated with catalase, and finally re-purified with Talon resin. Concentration and purity of recombinant CD148 was assessed by SDS-PAGE followed by staining with Coomassie Brilliant Blue. To reduce CD148, $5 \mu \mathrm{g}$ of pre-oxidized CD148 was treated with $90 \mu \mathrm{g} \mathrm{GST-}$ CSE bound to GSH agarose resin and $1 \mathrm{mM}$ cystine, GSH agarose resin and $1 \mathrm{mM}$ cystine, 1 $\mathrm{mM}$ cystine, $1 \mathrm{mM}$ cysteine, $10 \mathrm{mM}$ DTT or vehicle at $37^{\circ} \mathrm{C}$ for 30 minutes. Following incubation, the samples were centrifuged and the supernatants were transferred to a 96 well plate to assay for phosphatase activity.

Fluorimetric Detection of Phosphatase Activity: Samples were incubated with $30 \mu \mathrm{M}$ fluorescein diphosphate (FDP) in $100 \mu \mathrm{L}$. Immediately following the addition of FDP, phosphatase activity was measured every minute for $20 \mathrm{~min}$ at $37^{\circ} \mathrm{C}$ on a Synergy HT Multi-Mode Microplate Reader with 485/528 nm filters. Background fluorescence was subtracted out of all values presented.

\section{Results}


The Reaction of Cyanide ( $\mathrm{CN}^{-}$) with Hydropersulfides: The reaction of $\mathrm{CN}^{-}$with hydropersulfides to give thiocyanate ( $\mathrm{SCN}$ ) and the corresponding thiol is well established, [for example, 28,29] (Reaction 1). This reaction, sometimes referred to as cyanolysis, is mechanistically ambiguous [e.g. 11].

$$
\mathrm{RSSH}+\mathrm{CN}^{-} \rightarrow \mathrm{RSH}+\mathrm{SCN}^{-}
$$

In order to begin to examine the mechanisms/conditions of this reaction, the $\mathrm{pH}$-dependence of the reaction of $\mathrm{CN}^{-}$with 2-hydroxy-1-ethane hydropersulfide (HE-SSH) was examined. The approximate $\mathrm{pK}_{\mathrm{a}}$ of HE-SSH is 6-7 (hydropersulfides typically have $\mathrm{pK}_{\mathrm{a}}$ values of $1-2 \mathrm{pK}_{\mathrm{a}}$ units lower than the corresponding thiols [11]). Thus, at pH 10, HE-SS is the predominant form while at $\mathrm{pH} 7.4$ significant levels of the protonated HE-SSH species will be present. Also, $\mathrm{HCN}$ has a $\mathrm{pK}_{\mathrm{a}}$ of 9.2 so the predominant form of this species will be $\mathrm{CN}^{-}$at $\mathrm{pH} 10$. Previous work indicates that persulfide anions (RSS) absorb at $340 \mathrm{~nm}[10,30]$. As shown in Figure 1A the absorbance of HE-SS at $340 \mathrm{~nm}$ did not change appreciably for 2 minutes after the addition of 1 and $0.5 \mathrm{mM} \mathrm{CN}^{-}$at $\mathrm{pH} 10$, indicating that $\mathrm{HE}^{-S S}$ does not readily react with $\mathrm{CN}^{-}$. However, at $\mathrm{pH} 7.2$, the addition of 1 and $0.5 \mathrm{mM}$ levels of $\mathrm{CN}^{-}$to a HE-SSH/HE-SS solution shows a concentration-dependent and significant decrease in HE-SS absorbance, consistent with reaction of nucleophilic $\mathrm{CN}^{-}$with the protonated and electrophilic HE-SSH species (Figure 1B).
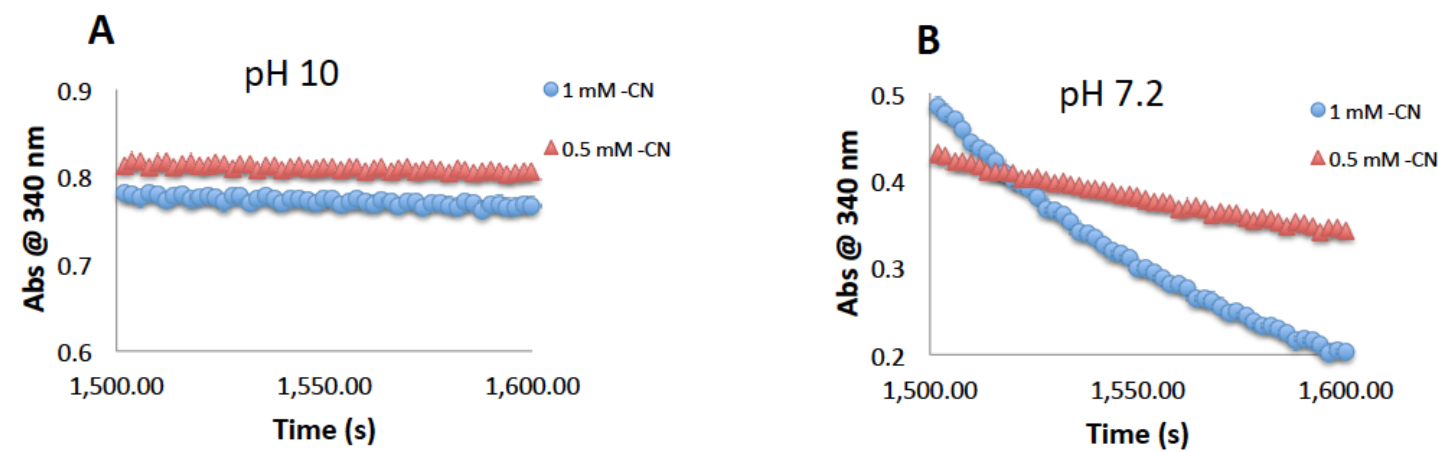

Figure 1: $\mathrm{pH}-$ Dependency of the reaction of $\mathrm{CN}^{-}$with HE-SSH. HE-SSH was made via reaction of HE-SS-HE with $\mathrm{H}_{2} \mathrm{~S}$ (see Materials and Methods section) and allowed to react for 1,500 seconds (25 min.) prior to the addition of $\mathrm{CN}^{-}$at the levels indicated. Levels of HE-SS/HE-SS-HE were monitored via HE-SS absorbance at $340 \mathrm{~nm}$. (A) $\mathrm{pH}$ 10, (B) $\mathrm{pH} 7.2$. 
Further experiments aimed at examining the reaction of $\mathrm{CN}^{-}$with $\mathrm{HE}-\mathrm{SSH}$ under a wider range of $\mathrm{pH}$ conditions were attempted. However, under more acidic conditions precipitates formed, likely due to proton-dependent side reactions and formation of polysulfides, including insoluble $\mathrm{S}_{8}$. Moreover, at lower $\mathrm{pH}$ values, the concentrations of $\mathrm{NaCN}$ required to give equilibrium concentrations of 1.0 and $0.5 \mathrm{mM} \mathrm{CN}^{-}\left(\mathrm{pK}_{\mathrm{a}}\right.$ of $\mathrm{HCN}$ is 9.2) were beyond the solubility of $\mathrm{NaCN}$. Thus, only a qualitative analysis of the effect of $\mathrm{pH}$ on this reaction can be performed at this time.

${ }^{1} \mathrm{H}$-NMR Analysis of the Reactions of Nucleophiles with Hydropersulfides: In an effort to further examine the reaction of nucleophiles with electrophilic hydropersulfides in aqueous solution, the reaction of a hydropersulfide with a variety of nucleophiles was examined using ${ }^{1} \mathrm{H}-\mathrm{NMR}$. In a recent report from the Nagy lab [31], it was demonstrated that hydropersulfides can be distinguished from other thiol species (i.e. thiols and disulfides) using ${ }^{1} \mathrm{H}$-NMR spectroscopy. The ${ }^{1} \mathrm{H}$-NMR chemical shifts for both the $\alpha$ - and $\beta$-protons for cysteine (Cys-SH), cystine (CysSS-Cys) and cysteine hydropersulfide (Cys-SSH) are easily distinguished from each other and therefore can be used to monitor reactions of these species. Thus, utilizing ${ }^{1} \mathrm{H}-\mathrm{NMR}$ to monitor the methylene protons alpha to the sulfur atom, the reactivity of various nucleophiles with the 2hydroxy-1-ethane thiol (HE-SH)-derived hydropersulfide was examined. When the disulfide (HE-SS-HE) was reacted with $\mathrm{H}_{2} \mathrm{~S}$ at pH 10, chemical shifts associated with $\mathrm{HE}-\mathrm{SH}$ and the corresponding persulfide, HE-SSH, were observed (formed via Reaction 2) (Figure 2A).

$$
\text { HE-SS-HE }+\mathrm{H}_{2} \mathrm{~S} \rightleftharpoons \mathrm{HE}-\mathrm{SSH}+\mathrm{HE}-\mathrm{SH}
$$



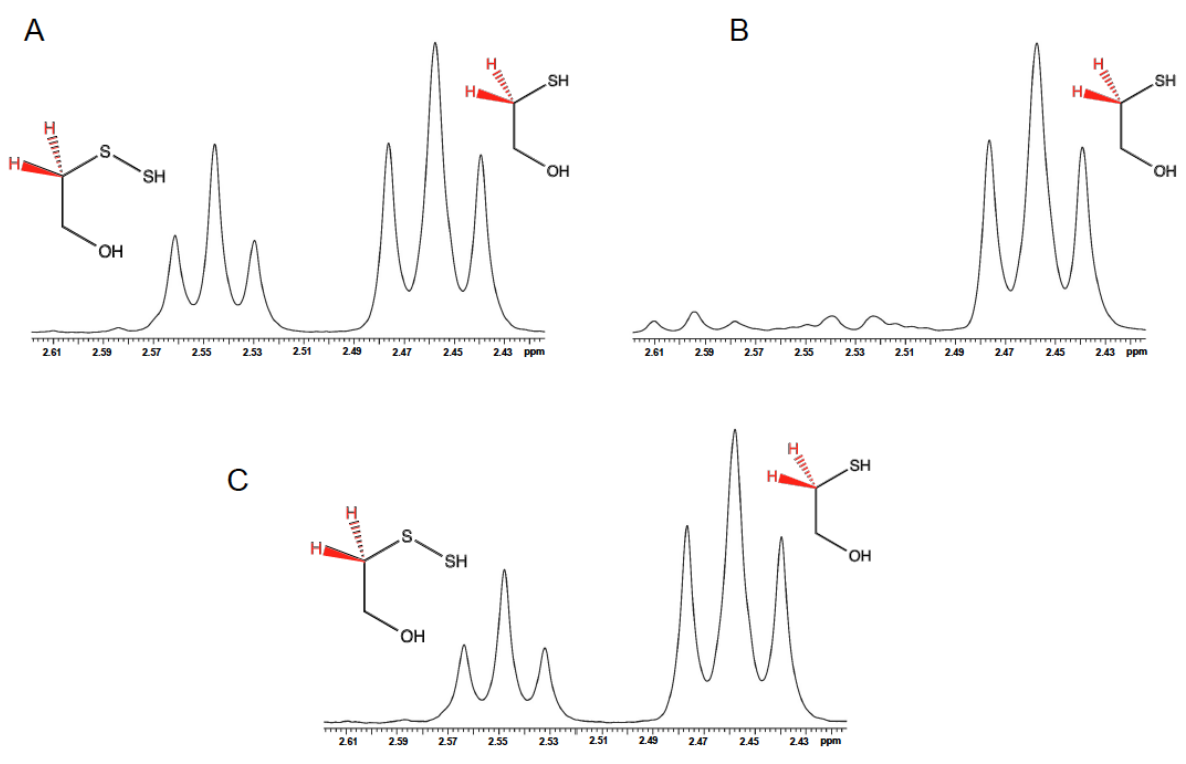

Figure 2: ${ }^{1} \mathrm{H}-\mathrm{NMR}$ Analysis of the reaction of $\mathrm{H}_{2} \mathrm{~S}$ with $\mathrm{HE}-\mathrm{SS}-\mathrm{HE}$ and subsequent reaction of $\mathrm{HE}-\mathrm{SSH}$ with nucleophiles. (A) ${ }^{1} \mathrm{H}-\mathrm{NMR}$ signals for methylene protons adjacent to the sulfur atoms (shown in red) for the products of the reaction of HE-SS$\mathrm{HE}$ with $\mathrm{NaSH}$ (HE-SSH and HE-SH). (B) Addition of $\mathrm{CN}^{-}$to the HE-SSH/HE-SH product mixture. (C) Addition of $\mathrm{N}_{3}{ }^{-}$to the $\mathrm{HE}-\mathrm{SSH} / \mathrm{HE}-\mathrm{SH}$ product mixture (note: same results observed for $\mathrm{NH}_{2} \mathrm{NH}_{2}$ and $\mathrm{NH}_{2} \mathrm{OH}$ ).

As shown in Figure 2B, addition of $\mathrm{CN}^{-}$to the reaction mixture at a 5-fold excess results in the loss of the ${ }^{1} \mathrm{H}$ resonance associated with HE-SSH (after 1 hour), consistent with Reaction 1 above. Addition of the phosphine tris(2-carboxyethyl)phosphine (TCEP) or the thiol dithiothreitol (DTT) also led to the loss of the HE-SSH species (data not shown). Thus, it is clear that ${ }^{1} \mathrm{H}$ NMR can be used to examine the reactivity of hydropersulfides with nucleophiles. The reaction of HE-SSH with other nucleophiles was also examined using this method. Interestingly, the nitrogen-based nucleophiles azide $\left(\mathrm{N}_{3}{ }^{-}\right)$, hydrazine $\left(\mathrm{H}_{2} \mathrm{NNH}_{2}\right)$ and hydroxylamine $\left(\mathrm{NH}_{2} \mathrm{OH}\right)$ all added at a 5-fold excess did not react with HE-SSH at either pH 10 or 7 (data not shown) under the conditions where $\mathrm{CN}^{-}$reactivity was observed (Figure $\mathbf{2 C}$ ).

\section{The Site of Nucleophilic Attack:}

Reactions of nucleophiles with electrophiles can be viewed as an interaction between the electrons in the highest-occupied molecular orbital (HOMO) of the nucleophile and the 
lowest-unoccupied molecular orbital of the electrophile [for example, 32]. In an attempt to evaluate the relative electrophilicity of the two sulfur atoms of RSSH, the LUMO of a hydropersulfide $\left(\mathrm{CH}_{3} \mathrm{SSH}\right)$ was calculated in a polar environment. As shown in Figure 3A, examination of the LUMO appears to indicate that attack at either sulfur atom is possible, since the lobes of the orbital on both sulfurs are large, although they are differently shaped. Examination of the electrostatic potential surface map for $\mathrm{CH}_{3} \mathrm{SSH}$ also seems consistent with possible nucleophilic attack at either sulfur, with possibly a slight preference for terminal sulfur attack (Figure 3B). These arguments are based on reactant properties, however, which do not always reflect kinetic behavior. The barrier and reaction energy for the established reaction of $\mathrm{CN}^{-}$with a hydropersulfide were also predicted and, as shown in Figure $\mathbf{3 C}$, the reaction of $\mathrm{CN}^{-}$ at the internal sulfur atom is kinetically favored (slightly) over attack at the terminal sulfur (11.9 $\mathrm{kcal} / \mathrm{mol}$ barrier for internal sulfur attack versus a $12.6 \mathrm{kcal} / \mathrm{mol}$ barrier for terminal sulfur attack). However, attack at the terminal sulfur atom is strongly favored thermodynamically by over 23 $\mathrm{kcal} / \mathrm{mol}$.

(a)

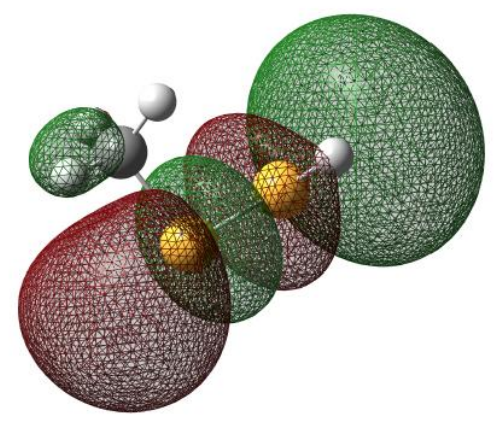

(b)

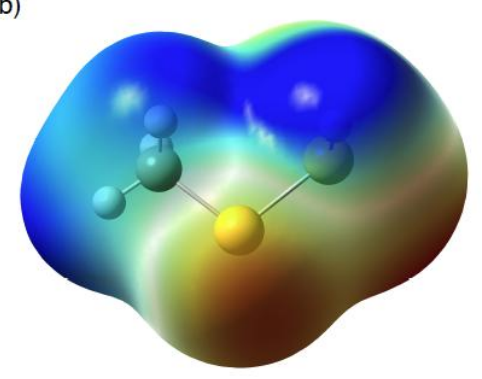

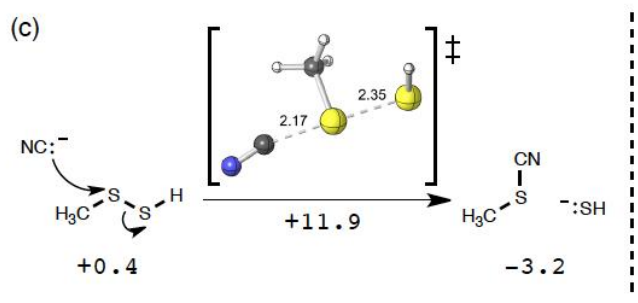

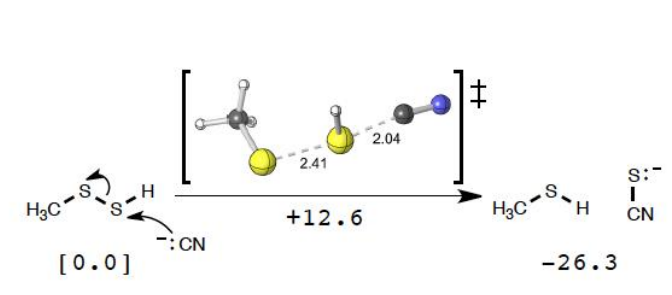

Figure 3: (A) LUMO for $\mathrm{CH}_{3}-\mathrm{SSH}$ (SMD(water)-M06-2X/6-31+G(d,p)). (B) Electrostatic potential surface for $\mathrm{CH}_{3}-\mathrm{SSH}$. (C) Calculated transition state structure and reaction free energies $(\mathrm{kcal} / \mathrm{mol})$ for the reaction of $\mathrm{CN}^{-}$with $\mathrm{CH}_{3}-\mathrm{SSH}$ at both sulfur atoms. 
The above examples involve reactions whereby the sulfur leaving group is anionic (i.e. an $\mathrm{RS}^{-}$leaving group for nucleophilic attack at the terminal sulfur atom or an $\mathrm{HS}^{-}$leaving group for nucleophilic attack at the internal sulfur atom). If the reaction involves a neutral leaving group (i.e. $\mathrm{RSH}$ or $\mathrm{H}_{2} \mathrm{~S}$ ), then the relative proton affinities of the two sulfur atoms will be an important consideration since protonation of the sulfur leaving group will be an important step prior to or concurrent with nucleophilic cleavage of the S-S bond. Determination of proton affinities for the two sulfur atoms in methyl hydropersulfide indicates that protonation on the internal sulfur atom is favored over protonation at the terminal sulfur $(239.9 \mathrm{kcal} / \mathrm{mol}$ versus $233.2 \mathrm{kcal} / \mathrm{mol}$, respectively). This would indicate that nucleophilic attack on the terminal sulfur atom would be favored when a neutral leaving group is possible.

\section{Reaction of GSSH with $\mathrm{O}_{2}$ :}

The above results confirm that hydropersulfides can be electrophilic, especially with softer nucleophiles such as $\mathrm{CN}^{-}$or phosphines. It is also reported that hydropersulfides can also be nucleophilic and reducing as well. Thus, these properties of hydropersulfides were also examined.

The reaction of the hydropersulfide GSSH with the oxidant $\mathrm{O}_{2}$ was examined first by simply determining the loss of the GSS absorbance at $340 \mathrm{nM}$ as a function of $\mathrm{O}_{2}$ levels. As shown in Figure 4A, HE-SS (at $\mathrm{pH}^{-10}$ ) is fairly stable under $\mathrm{N}_{2}$ with an extremely slow loss of absorbance at $340 \mathrm{~nm}$. However, in air the rate of loss of absorbance at $340 \mathrm{~nm}$ increases by 2.4-fold over reaction under $\mathrm{N}_{2}$. Under pure $\mathrm{O}_{2}$ the rate increases even further to 8.8-fold over reaction under $\mathrm{N}_{2}$. Thus, hydropersulfides react with $\mathrm{O}_{2}$. In order to compare the relative reactivities of hydropersulfides and thiols with $\mathrm{O}_{2}$, the relative rates of $\mathrm{O}_{2}$ consumption by these two species was determined. 

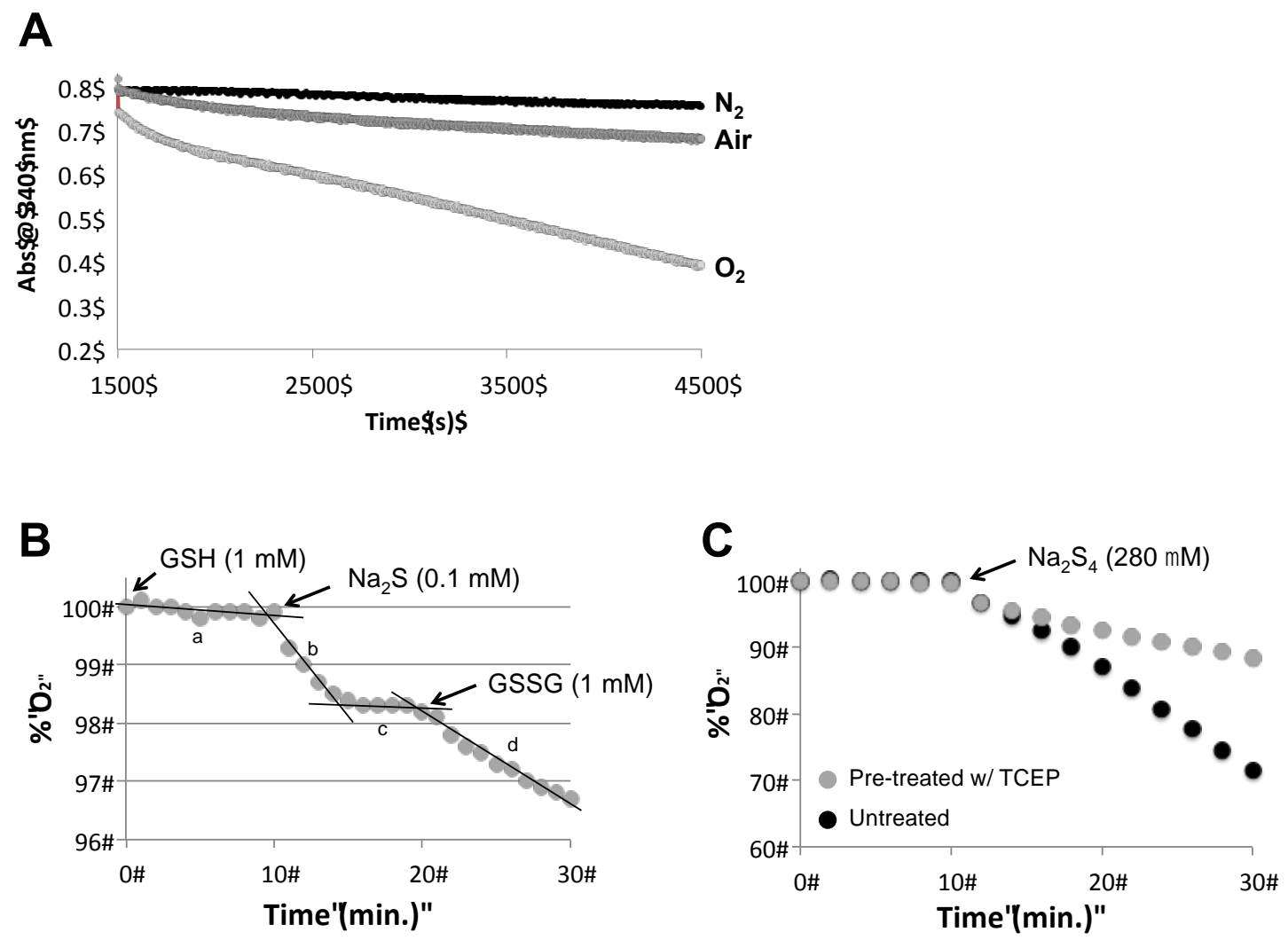

Figure 4: (A) Loss of HE-SS ${ }^{-}$absorbance at $340 \mathrm{~nm}$ under $\mathrm{N}_{2}$, air and $\mathrm{O}_{2}(\mathrm{pH} 10)$. note: the hydropersulfide forming reaction was run for $1500 \mathrm{~s}$ prior to allowing exposure to $\mathrm{N}_{2}$, air or $\mathrm{O}_{2}$. (B) Consumption of $\mathrm{O}_{2}$ (measured via a Clarke electrode) by sequential addition of $\mathrm{GSH}$ (slope $\mathbf{a}$, final conc. $1 \mathrm{mM}$ ), $\mathrm{Na}_{2} \mathrm{~S}$ solution (slope $\mathbf{b}$ and $\mathbf{c}$, final conc. 0.1 $\mathrm{mM}$ ) and GSSG (slope d, final conc. $1 \mathrm{mM}$ ). (C) $\mathrm{O}_{2}$ Consumption by commercial $\mathrm{Na}_{2} \mathrm{~S}_{4}$ with and without pre-treatment with TCEP-resin.

Using a Clarke electrode to monitor $\mathrm{O}_{2}$ consumption, the ability of various sulfurcontaining species to react with dissolved $\mathrm{O}_{2}$ was monitored. As shown in Figure 4B, the addition of GSH (1 mM final concentration) to a buffered aqueous solution ( $\mathrm{pH} 7.4$ ) does not cause a perceptible consumption of $\mathrm{O}_{2}$ (slope a), consistent with reported extremely small rate constants for this uncatalyzed reaction $\left(10^{-4}-10^{-3} \mathrm{M}^{-1} \mathrm{~s}^{-1}\right.$ under basic conditions) [33]. However, the addition of sodium sulfide $\left(\mathrm{Na}_{2} \mathrm{~S}\right)$ to a buffered solution containing $\mathrm{GSH}$ causes an immediate, relatively fast consumption of $\mathrm{O}_{2}$ (slope $\mathbf{b}$ ), followed by a slower, almost imperceptible rate of $\mathrm{O}_{2}$ consumption (slope c). (Note: the amount of $\mathrm{O}_{2}$ consumed represented by slope $\mathbf{b}$ is only a small percentage of the overall amount of $\mathrm{Na}_{2} \mathrm{~S}$ added). When $\mathrm{GSSG}$ is then added to solution, a relatively rapid rate of $\mathrm{O}_{2}$ consumption occurs (slope d). Interestingly, 
when the $\mathrm{Na}_{2} \mathrm{~S}$ solution is pretreated with resin-bound tris(2-carboxyethyl)phosphine (TCEP) (which can be removed from the treatment solution via centrifugation), the $\mathrm{O}_{2}$ consumption represented by slope $\mathbf{b}$ disappears (data not shown) and only the slow phase of $\mathrm{O}_{2}$ consumption remains (slope c). Also, it was found that the addition of the chelator diethylenediaminepentaacetic acid (DETAPAC) had no significant affect on these results, indicating that trace metal catalysis was not likely in these reactions (data not shown).

Since most commercial preparations of $\mathrm{Na}_{2} \mathrm{~S}$ contain polysulfide impurities $\left(\mathrm{Na}_{2} \mathrm{~S}_{n}, \mathrm{n}>1\right)$ [34] and phosphines are capable of reacting with and removing polysulfides (Reaction 3) $[28,35,36]$, it is likely that the elimination of the rapid $\mathrm{O}_{2}$-consuming process (slope $\mathbf{b}$ ) is due to the TCEP-mediated elimination of the trace $\mathrm{O}_{2}$-reactive polysulfides impurities.

$$
\mathrm{HS}_{\mathrm{n}} \mathrm{SH}+\mathrm{R}_{3} \mathrm{P}+\mathrm{H}_{2} \mathrm{O} \rightarrow \mathrm{R}_{3} \mathrm{PO}+\mathrm{H}_{2} \mathrm{~S}+\mathrm{HS}_{(\mathrm{n}-1)} \mathrm{SH} \rightarrow \text { etc. }
$$

The idea of enhanced $\mathrm{O}_{2}$ reactivity by polysulfides, compared to simple thiol or sulfide species, is further supported by the finding that the commercially available polysulfide disodium tetrasulfide $\left(\mathrm{Na}_{2} \mathrm{~S}_{4}\right)$ was found to react more quickly with $\mathrm{O}_{2}$ than $\mathrm{Na}_{2} \mathrm{~S}$. However, after treatment with TCEP (which should convert polysulfides to sulfides) or $\mathrm{CN}^{-}$(not shown) the reactivity with $\mathrm{O}_{2}$ was significantly attenuated (Figure $4 \mathrm{C}$ ).

The results above are consistent with the idea that perthiyl radical (RSS·) is more stable than the corresponding thiyl radical (RS·) both of which are the likely initial products from reaction with $\mathrm{O}_{2}$ (Reactions 4 and 5).

$$
\begin{aligned}
& \mathrm{RSS}^{-}+\mathrm{O}_{2} \rightarrow \text { RSS }+\mathrm{O}_{2}^{-\cdot} \\
& \mathrm{RS}^{-}+\mathrm{O}_{2} \rightarrow \text { RS. }+\mathrm{O}_{2}^{-\cdot}
\end{aligned}
$$

To further address this idea, the $\Delta \mathrm{Gs}$ for removal of a single electron from $\mathrm{CH}_{3} \mathrm{~S}^{-}$and $\mathrm{CH}_{3} \mathrm{SS}^{-}$to form $\mathrm{CH}_{3} \mathrm{~S}$. and $\mathrm{CH}_{3} \mathrm{SS}$. were calculated to be 87 and $79 \mathrm{kcal} / \mathrm{mol}$, respectively. Thus, the perthiyl anion is predicted to be more easily oxidized, by approximately $8 \mathrm{kcal} / \mathrm{mol}$, consistent with the finding that $\mathrm{RSS}^{-}$is a better reductant than $\mathrm{RS}^{-}$.

Persulfides as Biological Reductants: The above results indicate that hydropersulfides can react with $\mathrm{O}_{2}$, presumably serving as a one-electron reductant. The ability of hydropersulfides to reduce oxidized thiol proteins via presumed 2 -electron processes was also examined. Thus, 
using a well-characterized cysteine phosphatase, CD148 [37] as a model cysteine protein, the ability of cysteine Cys-SH, Cys-SS-Cys, Cys-SSH and dithiothreitol (DTT) to reduce oxidized protein back to an active reduced form was examined. As shown in Figure 5, inactive CD148 (pre-oxidized via treatment with $\mathrm{H}_{2} \mathrm{O}_{2}$ ) is readily reduced back to the active form using DTT. Since DTT is a non-physiological and highly reducing dithiol, this result serves as a positive control to indicate that the oxidized CD148 can be reduced back to the active thiol form.

Addition of Cys-SH to oxidized CD148, on the other hand, does not reduce the protein back to the active form over the course of the experiment. This is not surprising considering that the two-electron reduction potentials for oxidized DTT (oxDTT/redDTT, $\mathrm{E}^{\mathrm{o}^{\prime}}=-0.33 \mathrm{~V}$ [38]) and CysSS-Cys (Cys-SS-Cys/2Cys-SH,2H $\mathrm{H}^{+}, \mathrm{E}^{\mathrm{o}^{\prime}}=-0.21 \mathrm{~V}$ [38]) indicate that reduced DTT is a superior 2 electron reductant compared to Cys-SH. Expectedly, Cys-SS-Cys addition to oxidized and inactive CD148 showed no regeneration of activity. This is also not surprising since Cys-SSCys is an oxidized form of Cys-SH and will not act as a reductant. However, when the enzyme cystathionine gamma-lyase (CSE) is added to the Cys-SS-Cys/oxidized CD148 mixture, CD148 enzyme activity returns in a time-dependent fashion. As shown previously [3], CSE is capable of cleanly converting Cys-SS-Cys to Cys-SSH and pyruvate. Since pyruvate has no effect on this system (data not shown), Cys-SSH must be the reductant capable of facile reduction of oxidized CD148 to the active reduced form.

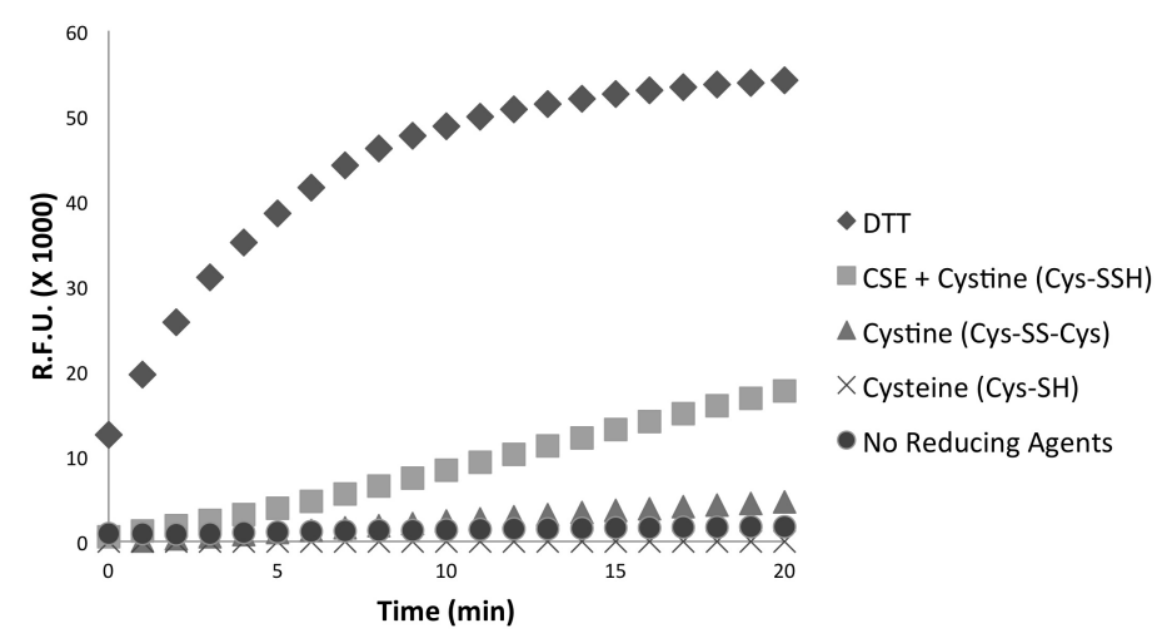

Figure 5: Recovery of oxidized $\left(\mathrm{H}_{2} \mathrm{O}_{2}\right) \mathrm{CD} 148$ enzyme activity by various reductants/treatments. Purified, pre-oxidized CD148 was treated with various reductants or controls for $30 \mathrm{~min}$. at $37^{\circ} \mathrm{C}$. After treatment, CD148 activity was assayed, using 
fluorescein diphosphate as a substrate. Fluorescence was measured every minute for $20 \mathrm{~min}$.

Theoretical view of the nucleophilicity of RSSH compared to RSH: The nucleophilicity of hydropersulfides (especially the persulfide anion) is proposed to be superior to that of the corresponding thiol. One metric for predicting relative nucleophilicity is via comparison of the energies of the HOMOs of the species in question [for example, 39]. Thus the relative energies of the HOMOs of a model persulfide versus the corresponding thiol were determined. The energy of the $\mathrm{HOMO}$ of $\mathrm{CH}_{3} \mathrm{~S}^{-}$was predicted to be $-133.7 \mathrm{kcal} / \mathrm{mol}$ whereas the energy of the $\mathrm{HOMO}$ of $\mathrm{CH}_{3} \mathrm{SS}^{-}$was predicted to be $-126.6 \mathrm{kcal} / \mathrm{mol}$. Thus, the $\mathrm{HOMO}$ of $\mathrm{CH}_{3} \mathrm{SS}^{-}$is about 7 $\mathrm{kcal} / \mathrm{mol}$ higher in energy than the $\mathrm{HOMO}$ of $\mathrm{CH}_{3} \mathrm{~S}^{-}$, consistent with the idea that RSS is more nucleophilic. (Interestingly, the relative HOMO energies are found to be reversed for the protonated species, $-183.6 \mathrm{kcal} / \mathrm{mol}$ for $\mathrm{CH}_{3} \mathrm{SH}$ and $-186.2 \mathrm{kcal} / \mathrm{mol}$ for $\mathrm{CH}_{3} \mathrm{SSH}$, although these energies are close).

\section{Discussion}

Hydropersulfides have been found to be present in mammalian tissues, cells and plasma with GSSH being the species in greatest abundance [3]. The presence of other hydropersulfides such as Cys-SSH as well as numerous protein persulfides are also reported. The prevalence of these species alludes to a possible biological function, which will undoubtedly be highly dependent on their chemical properties/reactivities. It has already been hypothesized that hydropersulfides possess unique chemical reactivity that is distinct from thiols and that these chemical properties may confer biological utility. For example, hydropersulfide generation may be a protective mechanism for cells exposed to oxidative or electrophilic stress or may enhance the reactivity of thiol proteins [11]. Cellular hydropersulfide formation can occur via the actions of the enzyme cystathionine gamma-lyase (CSE) acting on the substrate cystine (CysSS-Cys) generating Cys-SSH $[3,40]$ and Cys-SS-Cys uptake into cells is dependent on the transport protein $\mathrm{xCT}$ [41]. Interestingly, both CSE and $\mathrm{xCT}$ are inducible under oxidative and/or electrophilic stress $[42,43,44]$ indicating that hydropersulfide generation may be a response to these cellular stresses. In order to evaluate the plausibility of this idea, it is necessary to examine the chemistry of hydropersulfides to determine whether their reactivity/properties will allow them to serve this role.

Herein, it is found that electrophilic hydropersulfides (i.e. the protonated RSSH species and not the anionic RSS species) can react with nucleophiles at either sulfur atom. Reaction 
with $\mathrm{CN}^{-}$occurs with the protonated hydropersulfide and is thermodynamically favored to give products whereby the terminal, sulfane sulfur is transferred to $\mathrm{CN}^{-}$. The analogous cleavage of disulfides (RSSR) by $\mathrm{CN}^{-}$is a well-studied reaction [for example, 45-48] (Reaction 6).

$$
\mathrm{RSSR}^{\prime}+\mathrm{CN}^{-} \rightarrow \mathrm{RSCN}+\mathrm{R}^{\prime} \mathrm{S}^{-}
$$

This reaction is very slow, as evidenced by the low second order rate constants $\left(\leq 1 \mathrm{M}^{-1} \mathrm{~s}^{-1}\right.$ at $\mathrm{pH}$ 7.5 - 12.5) [49]. For unsymmetrical disulfides, the site of cyanide attack appears to be largely governed by the stability of the thiolate leaving group (i.e. the weakest thiolate base is generated) [46]. That is, reaction of $\mathrm{CN}^{-}$with unsymmetrical disulfides affords the most stable thiolate product and the corresponding alkyl thiocyanate. The generation of $\mathrm{SCN}$ from cyanolysis (Reaction 1) implies that $\mathrm{CN}^{-}$attacks the terminal sulfane ${ }^{1}$ sulfur (assuming a single step process, which is not necessarily the case and discussed below). Previous studies have examined the reaction of other nucleophiles with hydropersulfides and reported that many factors can control the site of nucleophilic attack with both sulfur atoms being possible targets [for example, 50,51,52]. More recently Bailey and coworkers [53] generated an organic hydropersulfide and examined its reaction with various nucleophiles and bases in methylene chloride. Their work indicated that reducing species can react with a hydropersulfide to liberate $\mathrm{H}_{2} \mathrm{~S}$ whereas basic species led to thiol formation and elemental sulfur generation. Regardless, the results presented herein clearly indicate that the nucleophilic $\mathrm{CN}^{-}$reacts with the protonated and electrophilic hydropersulfide (Figure 1) and although nucleophilic reaction can occur at either atom of a hydropersulfide, many factors seems to favor (albeit slightly) reaction at the terminal, sulfane sulfur atom in a purely chemical system.

It is evident from this work that only "soft" nucleophiles such as $\mathrm{CN}^{-}$, phosphines and thiols react readily with hydropersulfides whereas "hard(er)" nitrogen-based nucleophiles such as $\mathrm{NH}_{2} \mathrm{OH}, \mathrm{NH}_{2} \mathrm{NH}_{2}$ and $\mathrm{N}_{3}{ }^{-}$are relatively unreactive under the conditions of these experiments (for example, Figure 2). This is consistent with a "soft" electrophilic character associated with the S-S group of the hydropersulfide. Thus, the soft persulfide electrophile clearly favors reaction with soft nucleophiles. As alluded to above, hydropersulfides, $\mathrm{RSSH}$, and disulfides, RSSR, are clearly structurally and chemically analogous electrophiles. Importantly, studies on the nucleophilic cleavage of disulfides have also been examined previously and could provide some support for the conclusions/ideas presented in this study. Consistent with the results herein with hydropersulfides, cleavage of disulfides occurs readily with soft nucleophiles such as phosphines and thiols but is extremely difficult with hard bases/nucleophiles such as hydroxide 
ion or amines [for example, see 54]. Indeed, a systematic study of the ability of various nucleophiles to cleave disulfides revealed the following rank order for "S-nucleophilicity" to be $\mathrm{RS}^{-}>$phenyl-S $>\mathrm{CN}^{-}>\mathrm{SO}_{3}{ }^{-}>\mathrm{HO}^{-}>\mathrm{N}_{3}{ }^{-}>\mathrm{SCN}$ [45], an order that may be expected to hold for hydropersulfides as well.

The fact that the reaction of $\mathrm{CN}^{-}$with $\mathrm{RSSH}$ generates ${ }^{-} \mathrm{SCN}$ and $\mathrm{RSH}$ (Reaction 1) would appear to indicate that the site of nucleophilic attack is the terminal, sulfane sulfur of the hydropersulfide. However, previous work indicates that nucleophilic attack at the internal sulfur atom is also possible [for example, 50-53] (Reaction 7).

$$
\mathrm{Nuc}^{-}+\mathrm{RSSH} \rightarrow \mathrm{Nuc}-\mathrm{SR}+\mathrm{HS}^{-}
$$

Indeed, based on the relative $\mathrm{pK}_{\mathrm{a}}$ values for $\mathrm{RSH}$ (pKa approx. 8-10) versus $\mathrm{H}_{2} \mathrm{~S}$ ( $\mathrm{pK}$ approx. 7), which would make $\mathrm{HS}^{-}$a better leaving group than $\mathrm{RS}^{-}$, it would be expected that nucleophilic attack on RSSH would prefer the internal sulfur atom when there is an anionic leaving group. Computational evaluation of this question indicates that attack at either major lobe of the S-S $\sigma^{*}$ orbital by a nucleophile appears reasonable (although there appears to be a slight preference for attack at the terminal, sulfane sulfur atom) (Figure 3A,B). Attack at the terminal sulfur atom may be preferred based on sterics since the internal sulfur atom can be blocked by a large appended alkyl group. The above discussion assumes that the electrophilic species is neutral (i.e., RSSH) and that the leaving group is anionic (RS- or HS ${ }^{-}$). However, it needs to be considered that the electrophile could be the protonated species (i.e., $\mathrm{RS}(\mathrm{H}) \mathrm{SH}^{+}$or $\mathrm{RSSH}_{2}{ }^{+}$) allowing a neutral leaving group $\left(\mathrm{RSH}\right.$ or $\left.\mathrm{H}_{2} \mathrm{~S}\right)$ when attacked by a nucleophile. If this were the case, the preferred site of protonation will largely govern the site of nucleophilic attack (with attack occurring on the sulfur atom that is not protonated). Computational studies indicate that the internal sulfur atom is more basic than the terminal sulfur atom, predicting that protondependent cleavage of the S-S bond should primarily involve attack of the nucleophile at the terminal sulfur atom (Reaction 8).

$$
\mathrm{RS}^{+}(\mathrm{H}) \mathrm{SH}+\mathrm{Nuc}^{-} \rightarrow \mathrm{RSH}+\mathrm{Nuc}-\mathrm{SH}
$$

Overall, as has been noted by others (vide supra), nucleophilic attack at either sulfur atom of a hydropersulfide seems possible and it is likely that in biological systems (i.e. for protein-SSH species) attack at either sulfur atom can be governed by sterics, availability of 
protons, or other protein-derived effects. However, the above results seem to indicate that in a purely chemical system, attack at the terminal sulfur atom is slightly preferable.

Computational studies on the thermodynamics of the hydropersulfide- $\mathrm{CN}^{-}$reaction indicate that the kinetic and thermodynamic products are different (Figure $3 \mathbf{C}$ ) with attack at the internal sulfur atom being kinetically favored and attack at the terminal sulfur atom being thermodynamically favored. Therefore, we predict that reaction of $\mathrm{CN}^{-}$with the internal sulfur is reversible (reverse barrier of only $15 \mathrm{kcal} / \mathrm{mol}$ ), but reaction with the terminal sulfur is not (reverse barrier of nearly $40 \mathrm{kcal} / \mathrm{mol}$ ), being driven forward by proton transfer to form ${ }^{-} \mathrm{SCN}$ and thiol. Alternatively, reaction of $\mathrm{HS}^{-}$with the alkyl thiocyanate (RS-CN) species derived from internal attack can occur at the nitrile carbon, again resulting in the thermodynamically favored products 'SCN and RSH (Reaction 9).

$$
\mathrm{RS}-\mathrm{CN}+\mathrm{HS}^{-} \rightarrow \mathrm{RSH}+{ }^{-} \mathrm{SCN}
$$

Regardless, the thermodynamic products for this reaction are assuredly those where there is transfer of the terminal sulfur atom to the cyanide ion.

$\mathrm{O}_{2}$ is a poor thermodynamic one-electron oxidant $\left(\mathrm{O}_{2} / \mathrm{O}_{2} \cdot-\right.$ redox couple is $-0.33 \mathrm{~V}$, vs $\mathrm{NHE}, 1$ atm $\mathrm{O}_{2}, \mathrm{pH} 7$ ) thus generally prohibiting facile direct, non-catalytic, one-electron oxidation reactions with thiols. However, it is known that thiols can oxidize in aerobic environments (albeit very slowly). Importantly, hydropersulfides are considered to be superior reducing agents compared to their thiol counterparts [55]. For example, previous work indicates that persulfides are potent reductants, capable of easily reducing ferric cytochrome $c$ [10] reacting with and quenching radical species [54] and reacting with hydrogen peroxide [3] much more readily than their thiol counterparts. The results herein indicate that hydropersulfides can react with $\mathrm{O}_{2}$ (Figure 4A) more readily than its thiol counterpart (Figure 4B). These results are consistent with the idea that GSH does not readily react with $\mathrm{O}_{2}$ (Figure 4B, slope a) at a significant rate whereas formation of GSSH via Reaction 10 leads to a more rapid reaction with $\mathrm{O}_{2}$ (Figure 4B, slope d) (Reaction 11). The biphasic effect of $\mathrm{O}_{2}$ consumption observed for $\mathrm{Na}_{2} \mathrm{~S}$ addition (Figure 4B, slopes $\mathbf{b}$ and $\mathbf{c}$ ) can be attributed to polysulfide impurities in the $\mathrm{Na}_{2} \mathrm{~S}$ [34], which rapidly react with $\mathrm{O}_{2}$ (Reaction 12) since pretreatment of $\mathrm{Na}_{2} \mathrm{~S}$ with TCEP immobilized resin (known to react with sulfane sulfur species leading to fully reduced sulfides $[28,35,36]$ ) (Reaction 3 ) led to a presumed loss of contaminating polysulfide impurities and consequent loss of the rapid initial phase (Figure 4B, slope b) of $\mathrm{O}_{2}$ consumption, leaving only slope c. 


$$
\begin{aligned}
& \mathrm{GSSG}+\mathrm{HS}^{-} \rightarrow \mathrm{GSS}^{-}+\mathrm{GSH} \\
& \text { GSS }^{-}+\mathrm{O}_{2} \rightarrow \text { GSS. }+\mathrm{O}_{2}{ }^{\cdot-} \\
& \mathrm{SS}_{\mathrm{n}} \mathrm{S}^{-}+\mathrm{O}_{2} \rightarrow \mathrm{SS}_{\mathrm{n}} \mathrm{S} \cdot+\mathrm{O}_{2}{ }^{\cdot-}
\end{aligned}
$$

The utility of the TCEP-resin in this type of experiment is due to the fact that it can rapidly reduce and eliminate contaminating polysulfide species in sulfide solutions and then be easily removed from a sulfide solution via either centrifugation or filtration, therefore preventing TCEP interference in subsequent experiments.

By analogy with proposed reactions of thiols with $\mathrm{O}_{2}$ [for example, 56,57], it seems likely that $\mathrm{O}_{2}$ is acting as a one-electron oxidant generating the radical sulfur species (RSS.) and $\mathrm{O}_{2}{ }^{-*}$, as shown in Reaction 11). This is likely since RSS is known to be a superior reducing agent compared to the corresponding thiol [55]. The fate of either RSS - or $\mathrm{O}_{2}{ }^{-1}$ in this system is unknown and will undoubtedly be a function of reaction conditions (i.e. $\mathrm{pH}$, concentration, the availability of other reactants, etc.). The subsequent reactions of these intermediates are subjects of ongoing work.

The results from the $\mathrm{O}_{2}$-consumption experiments provide an approximate and maximum rate constant for the reaction of $\mathrm{GSSH}$ with $\mathrm{O}_{2}(\mathrm{pH} 7.0)$ under the conditions of this experiment of $0.3-0.4 \mathrm{M}^{-1} \mathrm{~s}^{-1}$ (assuming a concentration of $0.1 \mathrm{mM} \mathrm{GSSH}$, approximately 0.2 $\mathrm{mM} \mathrm{O}_{2}$ and an $\mathrm{O}_{2}$ consumption rate of approximately $7 \times 10^{-9} \mathrm{M} / \mathrm{s}$ ). It needs to be mentioned, however, that this value is only valid assuming the reacton of GSSH with $\mathrm{O}_{2}$ is analogous to the proposed reaction of $\mathrm{GSH}$ with $\mathrm{O}_{2}$ (i.e. overall second order reaction generating $\mathrm{O}_{2}{ }^{--}$and the corresponding sulfur-based radical). Although this reaction is significantly faster than the equivalent reaction of the corresponding thiol (compare with a $10^{-3}-10^{-4} \mathrm{M}^{-1} \mathrm{~s}^{-1}$ rate constant for the reaction of $\mathrm{RS}^{-}$with $\mathrm{O}_{2}$ [33]), this is still a slow reaction and likely not relevant under most biological conditions. However, there remains the possibility that catalytic $\mathrm{O}_{2}$-mediated oxidation of hydropersulfides may be relevant. Previous reports indicate that thiols are readily oxidized by $\mathrm{O}_{2}$ in the presence of metals [for example, 58,59]. It would not be surprising to expect that $\mathrm{O}_{2}$-mediated oxidation of persulfides would behave similarly. That is, the addition of redox metals could accelerate the reaction between $\mathrm{O}_{2}$ and persulfide. This is indeed found to be the case. Addition of catalytic levels of the cuprous acetonitrile complex $\left(\mathrm{Cu}^{\prime}\left(\mathrm{CH}_{3} \mathrm{CN}\right)_{4}^{+}\right)$to a solution of GSSH rapidly increased the rate of $\mathrm{O}_{2}$ consumption (data not shown). 
The above results are consistent with the idea that perthiyl radical (RSS·) is more stable than the corresponding thiyl radical (RS.) (assuming RSS. is the product after initial reaction with $\mathrm{O}_{2}$ (Reaction 9)). This is not unexpected since there is resonance stabilization of the odd electron associated with RSS $[11,60]$. Herein it is found that the persulfide anion is predicted to be more easily oxidized compared to the corresponding thiolate, by approximately $8 \mathrm{kcal} / \mathrm{mol}$, consistent with the finding that $\mathrm{RSS}^{-}$is a better reductant than $\mathrm{RS}^{-}$. Expectedly, the S-H bond dissociation energies (BDE) for RS-H and RSS-H are also significantly different: The BDE for the RS-H bond is reported to be approximately $92 \mathrm{kcal} / \mathrm{mol}$ and the BDE for RSS-H is reported to be only $70 \mathrm{kcal} / \mathrm{mol}$ [60]. These results indicate that $\mathrm{RSS}^{-}$is a better one-electron reductant than $\mathrm{RS}^{-}$and, correspondingly, RSSH is a better hydrogen atom donor compared to RSH.

Hydropersulfides are capable of facile reduction of oxidized thiols (or thiol-containing proteins) indicating that hydropersulfides are also good two-electron reductants (superior to thiols) [10]. This idea was further supported by the finding that hydropersulfides greatly increase the rate of CD148 activity restoration after enzyme oxidation by $\mathrm{H}_{2} \mathrm{O}_{2}$ (Figure 5). Combined with the previously discussed results, hydropersulfides appear to be superior reductants compared to thiols in both one- and two-electron processes. Loss of enzymatic activity of CD148 via treatment with $\mathrm{H}_{2} \mathrm{O}_{2}$ is undoubtedly the result of oxidation of the active-site cysteine thiol as has been seen in other similar phosphatases [for example, 61]. Oxidation of CD148 by $\mathrm{H}_{2} \mathrm{O}_{2}$ can theoretically lead to loss of activity via generation of an active site sulfenic acid (RSOH), disulfide (RSSR) or cyclic sulfenylamide species (protein backbone amide nitrogen bonded to the oxidized sulfur atom to make an isothiazolidinone) $[62,63]$. Since treatment of oxidized CD148 with DTT restores enzyme activity, it is presumed that further oxidized species such as sulfinic $(\mathrm{RS}(\mathrm{O}) \mathrm{OH})$ or sulfonic $\left(\mathrm{RS}(\mathrm{O})_{2} \mathrm{OH}\right)$ acids are not formed appreciably under the conditions of this experiment as DTT will not reduce sulfinic or sulfonic acids back to thiols. Figure 6 shows a possible mechanism of Cys-SSH-mediated reduction of oxidized CD148 back to the active thiol form. This mechanism depicts the inhibited CD148 as being a sulfenic acid, disulfide or sulfenylamide, all of which can be reduced to the corresponding thiol under biological conditions [for example, 64]. 


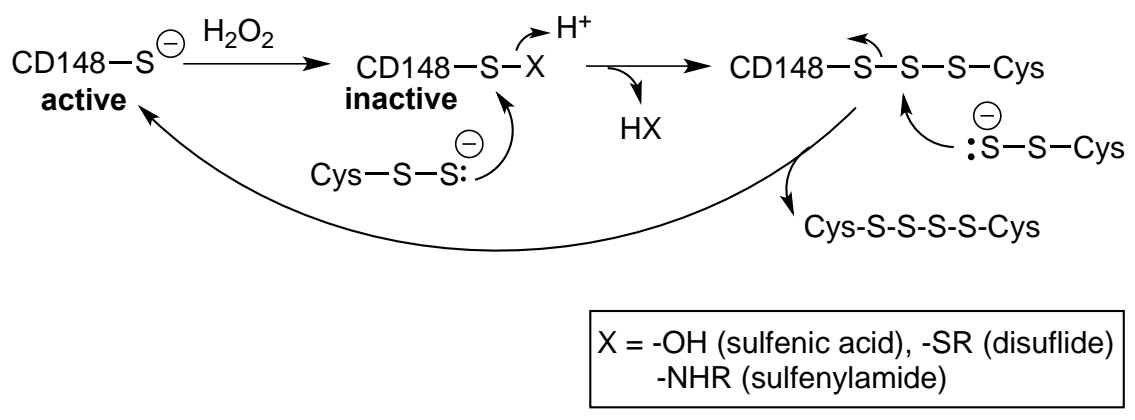

Figure 6: Possible mechanism of reduction of oxidized CD148 by a persulfide to the active thiol form.

From a mechanistic perspective, the ability of persulfides to readily reduce an oxidized thiol (i.e. sulfenic acid or disulfide) is most likely due, at least in part, to its superior nucleophilicity compared to the corresponding thiol. It has been reported that hydropersulfides are good nucleophiles, as evidenced by their ability to be trapped by electrophilic species [for example, 3,6,10]. Indeed, all steps depicted in Figure 6 rely on the nucleophilicity of the persulfide anion and results presented here are consistent with the idea that persulfides are superior to thiols as nucleophilic reductants.

It needs to be noted that a ranking of nucleophiles is not absolute and often depends on the nature of the electrophile, solvent and other reaction factors [for example, 65, 66]. The claim of increased nucleophilicity for RSSH/RSS over RSH/RS', in the context of this paper, refers to simple biochemical reactions with typical electrophiles known to react with thiols in aqueous solution (e.g., peroxides, electrophilic nitrogen oxides, oxidized sulfur species, alphabeta unsaturated carbonyls, alkyl halides, etc.). Although many factors may contribute to nucleophilicity, one significant factor is the energy of the HOMO of the nucleophile [for example, 38]. As mentioned above, reactions involving a nucleophile and an electrophile can be viewed as an interaction between the HOMO of the nucleophile and the LUMO of the electrophile [for example, 32]. The calculated energy of the HOMO for a hydropersulfide is approximately 7 $\mathrm{kcal} / \mathrm{mol}$ higher than that of the corresponding thiol, indicating a significant difference in nucleophilicity. In conjunction with having higher HOMO energies, free hydropersulfides are also more acidic than the corresponding free thiols by one to two $\mathrm{pK}_{\mathrm{a}}$ units [11] (indicating a higher concentration of the anionic species for persulfides by 10-100 times). Taken together, these facts are consistent with the prediction that free persulfides will often act as better nucleophiles in aqueous solution compared to their free thiol counterparts. Of course, the 
reactivity of thiols (and likely hydropersulfides) can be heavily influenced by protein environments, thus the relative reactivities of thiols versus hydropersulfides in proteins may not always follow this trend.

\section{Summary}

All of the above results are consistent with the idea that hydropersulfides can perform some of the same chemistry as thiols (i.e. one- or two-electron reduction and nucleophilic chemistry) as well as some chemistry associated with disulfides (i.e. act as an electrophile). That is, depending on the protonation state and conditions, hydropersulfides can be either nucleophilic or electrophilic. On the other hand, thiols can be nucleophilic and disulfides can be electrophilic, but both properties are not generally present in either species alone. This distinction may be important since the chemistry of a biological hydropersulfide can be either nucleophilic or electrophilic without requiring a change in oxidation state (as is the case with thiols/disulfides). Moreover, as a reductant and nucleophile, it appears that hydropersulfides are superior to thiols, a property that is not unexpected $[11,55]$. With regards to the reducing and nucleophilic nature of hydropersulfides, it is also worth noting that biological hydropersulfides are likely formed under cellular conditions of oxidative and/or electrophilic stress (vide supra) (afterall hydropersulfides are oxidized compared to a thiol), which indicates that a very reducing/nucleophilic species (persulfide anion) is only formed under oxidizing/electrophilic stress conditions. It is intriguing to speculate that this effect (i.e., the generation of a potent reductant/nucleophile only under oxidizing/electrophilic conditions) represents a protective response to cellular oxidative stress and/or electrophilic stress. However, whether these aspects of hydropersulfide chemistry are important to the putative biological utility of these species remains to be determined.

${ }^{1}$ The term sulfane sulfur is used to denote divalent sulfur bound sulfur atoms (i.e. the bolded sulfur atoms in the following structures: RSSH/RSS', RSSSR, RSSSSR, etc.)

Acknowledgements: This work was supported by grants from the National Institutes of Health (HL106598) and the National Science Foundation (CHE-1148641) to JMF, an MRI grant from the National Science Foundation (CHE-1126482) to the Sonoma State University Chemistry 
Department and an ACS-PRF grant (52801-ND4) to DJT. Computational support from the NSF's XSEDE program is also gratefully acknowledged 


\section{References}

1. Kajimura, M., Fukuda, R., Bateman, R. M., Yamamoto, T. and Suematsu, M. (2010) Interactions of multiple gas-transducing systems: Hallmarks and uncertainties of $\mathrm{CO}, \mathrm{NO}$ and $\mathrm{H}_{2} \mathrm{~S}$ gas biology, Antiox. Redox Signaling, 13, 157-192.

2. Wang, R. (2011) Physiological implications of hydrogen sulfide: A whiff exploration that blossomed, Physiol. Rev., 92, 791-896.

3. Ida, T., Sawa, T., Ihara, H., Kasamatsu, S., Kunieda, K., Tsuchiya, Y., Watanabe, Y., Kumagai, Y., Nishida, M., Suematsu, M., Motohashi, H., Fujii, S., Matsunaga, T., Yamamoto, M., Ono, K., Devarie-Baez, N. O., Xian, M., Fukuto, J. M. and Akaiake, T. (2014) Reactive cysteine persulfides and S-polysulfuration regulate oxidative stress and redox signaling, Proc. Natl. Acad. Sci., U. S. A., 111, 7606-7611.

4. Wright, C. M., Christman, G. D., Snellinger, A. M., Johnston, M. V. and Mueller, E. G. (2006) Direct evidence for the enzyme persulfide and disulfide intermediates during 4thiouridine biosynthesis, Chem. Commun., 3104-3106.

5. Souness, R. J., Kleffmann, T., Tchesnokov, E. P., Wilbanks, S. M., Jameson, G. B. and Jameson, G. N. L. (2013) Mechanistic implication of persulfenate and persulfide binding in the active site of cysteine dioxygenase, Biochemistry, 52, 7606-7617.

6. Pan, J. and Carroll, K. S. (2013) Persulfide reactivity in the detection of protein Ssulfhydration, ACS Chem. Biol., 8, 1110-1116.

7. Kimura, Y., Mikami, Y., Osumi, K., Tsugane, M., Oka, J. and Kimura, H. (2013) Polysulfides are possible $\mathrm{H}_{2} \mathrm{~S}$-derived signaling molecules in rat brain, FASEB J., 27, 2451 2457.

8. Sen, N., Paul, B. D., Gadalla, M. M., Mustafa, A. K., Sen, T., Xu, R., Kim, S. and Snyder, S. H. (2012) Hydrogen sulfide-linked sulfhydration of NFkB mediates its antiapoptotic actions, Mol. Cell, 45, 13-24. 
9. Mustafa, A. K., Gadalla, M. M., Sen, N., Kim, S., Mu, W., Gazi, S. K., Barrow, R. K., Yang, G., Wang, R. and Snyder, S. H. (2009) $\mathrm{H}_{2} \mathrm{~S}$ Signals through protein S-sulfhydration, Sci. Signaling, 2, 1-7.

10. Francoleon, N. E., Carrington, S. J. and Fukuto, J. M. (2011) The reaction of $\mathrm{H}_{2} \mathrm{~S}$ with oxidized thiols: Generation of persulfides and implications to $\mathrm{H}_{2} \mathrm{~S}$ biology, Arch. Biochem. Biophys., 516, 146-154.

11. Ono, K., Akaike, T., Sawa, T., Kumagai, Y., Wink, D. A., Tantillo, D. J., Hobbs, A. J., Nagy, P., Xian, M., Lin, J. and Fukuto, J. M. (2014) The redox chemistry and chemical biology of $\mathrm{H}_{2} \mathrm{~S}$, hydropersulfides and derived species: Implications of their possible biological activity and utility, Free Radic. Biol. Med., 77, 82-94.

12. Massey, V. and Edmondson, D. (1970) On the mechanism of inactivation of xanthine oxidase by cyanide, J. Biol. Chem., 245, 6595-6598.

13. Edmondson, D., Massey, V., Palmer, G., Beacham, L. M. III and Elion, G. B. (1972) The resolution of active and inactive xanthine oxidase by affinity chromatography, J. Biol. Chem., $247,1597-1604$.

14. Branzoli, U. and Massey, V. (1974) Evidence for an active site persulfide residue in rabbit liver aldehyde oxidase, J. Biol. Chem., 249, 4346-4349.

15. Agro, A. F., Mavelli, I., Cannella, C. and Federici, G. (1976) Activation of porcine heart mitochondrial malate dehydrogenase by zero valence sulfur and rhodanese, Biochem. Biophys. Res. Commun., 68, 553-560.

16. Bonomi, F., Pagani, S., Cerletti, P. and Cannella, C. (1977) Rhodanese-mediated sulfur transfer to succinate dehydrogenase, Eur. J. Biochem., 72, 17-24.

17. Hargrove, J. L. (1988) Persulfide generated from L-cysteine inactivates tyrosine aminotransferase, J. Biol. Chem., 263, 17262-17269. 
18. Fukuto, J. M., Carrington, S. J., Tantillo, D. J., Harrison, J. G, Ignarro, L. J., Freeman, B. A., Chen, A. and Wink, D. A. (2012) Small molecule signaling agents: The integrated chemistry and biochemistry of nitrogen oxides, oxides of carbon, dioxygen, hydrogen sulfide and their derived species, Chem. Res Toxicol., 25, 769-793.

19. Hemmerich, P. and Sigwart, C. (1963) $\mathrm{Cu}\left(\mathrm{CH}_{3} \mathrm{CN}\right)_{2}{ }^{+}$, ein Mittel zum Studium homogener Reaktionen des einwertigen Kupfers in wässriger Lösung. Experientia 19, 488-489.

20. Marenich, A. V., Cramer, C. J. and Truhlar, D. G. (2009) “Universal solvation model based on solute electron density and a continuum model of the solvent defined by the bulk dielectric constant and atomic surface tensions," J. Phys. Chem. B, 113, 6378-96.

21. Zhao, Y. and Truhlar, D. G. (2008) The M06 suite of density functionals for main group thermochemistry, thermochemical kinetics, noncovalent interactions, excited states, and transition elements: two new functionals and systematic testing of four M06-class functionals and 12 other functionals, Theor. Chem. Acc., 120, 215-41.

22. Gaussian 09, Revision B.01; Gaussian, Inc.: Wallingford, CT, 2004

23. Fukui, K. (1981) "The path of chemical-reactions - The IRC approach," Acc. Chem. Res., 14, 363-68.

24. Hratchian, H. P. and Schlegel, H. B. in Theory and Applications of Computational Chemistry: The First 40 Years, Ed. C. E. Dykstra, G. Frenking, K. S. Kim, and G. Scuseria (Elsevier, Amsterdam, 2005) 195-249.

25. Becke, A. D. (1988), "Density-functional exchange-energy approximation with correct asymptotic-behavior," Phys. Rev. A, 38, 3098-100.

26. Lee, C., Yang, W. and Parr, R. G. (1988), "Development of the Colle-Salvetti correlationenergy formula into a functional of the electron density," Phys. Rev. B, 37, 785-89. 
27. Miehlich, B., Savin, A., Stoll, H. and Preuss, H. (1989), "Results obtained with the correlation-energy density functionals of Becke and Lee, Yang and Parr," Chem. Phys. Lett., 157, 200-06.

28. Cavallini, D., Federici, G. and Barboni, E. (1970) Interaction of proteins with sulfide, Eur. J. Biochem., 14, 169-174.

29. Flavin, M. (1962) Microbial transsulfuration: The mechanism of an enzymatic disulfide elimination reaction, J. Biol. Chem., 237, 768-777.

30. Rao, G. S. and Gorin, G. (1959) Reaction of cystine with sodium sulfide in sodium hydroxide solution, J. Org. Chem., 24, 749-753.

31. Vasas, A., Doka, E., Fabian, I. and Nagy, P. (2014) Kinetic and Thermodynamic studies on the disulfide-bond reducing potential of hydrogen sulfide, Nitric Oxide, doi: 10.1016/j.niox.2014.12.003

32. Jaramillo, P., Domingo, L. R., Chamorro, E. and Perez, P. (2008) A further exploration of a nucleophilicity index based on the gas-phase ionization potentials, J. Mol. Struct.: THEOCHEM, 865, 68-72.

33. Bagiyan, G. A., Koroleva, I. K., Soroka, N.V. and Ufimtsev, A. V. (2003) Oxidation of thiol compounds by molecular oxygen in aqueous solutions, Russ. Chem. Bull., Int. Ed., 52, 11351141.

34. Greiner, R., Palinkas, Z., Basell, K., Becher, D., Antelmann, H., Nagy, P. and Dick, T. (2013) Polysulfides link $\mathrm{H}_{2} \mathrm{~S}$ to protein thiol oxidation, Antiox. Redox Signaling, 19, 1749-1765.

35. Ruegg, U. T. and Rudinger, J. (1977) Reductive cleavage of cystine disulfides with tributylphosphine, Methods Enzymol., 47, 111-116.

36. Shen, X., Peter, E. A., Bir, S., Wang, R. and Kevil, C. G. (2012) Analytical measurements of discrete hydrogen sulfide pools in biological specimens, Free Radical Biol. Med., 52, 2276-2283. 
37. Hermiston, M. L., Zikherman, J. and Zhu, J. W. (2009) CD45, CD148, and Lyp/Pep: Critical phosphatases regulating Src family kinase signaling networks in immune cells, Immunol. Rev., 228, 288-311.

38. Cleland, W. W. (1964) Dithiothreitol, a new protective reagent for SH groups, Biochemistry, 3, 480-482.

39. Bento, A. P. and Bickelhaupt, F. M. (2008) Nucleophilicity and leaving group ability in frontside and backside $\mathrm{S}_{\mathrm{N}} 2$ reactions, J. Org. Chem., 73, 7290-7299.

40. Stipanuk, M. H. (1986) Metabolism of sulfur-containing amino acids, Annu. Rev. Nutr., 6, 179-209.

41. Conrad, M. and Sato, H. (2012) The oxidative stress-inducible cystine/glutamate antiporter, system $X_{c}^{-}$: Cystine supplier and beyond, Amino Acids, 42, 231-246.

42. Wang, M., Guo, Z. and Wang, S. (2013) Cystathionine gamma-lyase expression is regulated by exogenous hydrogen peroxide in mammalian cells, Gene Expression, 15, 235-241.

43. Fox, B., Schantz, J.-T., Haigh, R., Wood, E., Moore, P. K., Viner, N., Spencer, J. P. E., Winyard, P. G. and Whiteman, M. (2012) Inducible hydrogen sulfide synthesis in chondrocytes and mesenchymal progenitor cells: Is $\mathrm{H}_{2} \mathrm{~S}$ a novel cytoprotective mediator in the inflamed joint?, J. Cell. Mol. Med. 16, 896-910.

44. Lewerenz, J., Hewett, S. J., Huang, Y., Lambros, M., Gout, P. W., Kalivas, P. W., Massie, A., Smolders, I., Methner, A., Pergande, M., Smith, S. B., Ganapathy, V. and Maher, P. (2013) The cystine/glutamate antiporter system $x_{c}{ }^{-}$in health and disease: From molecular mechanisms to novel therapeutic opportunities, Antiox. Redox Signaling, 18, 522-555.

45. Parker, A. J. and Kharasch, N. (1959) The scission of the sulfur-sulfur bond, Chem. Rev., 59, 583-628. 
46. Hiskey, R. G. and Carroll, F. I. (1961) Chemistry of aliphatic disulfides. II. Cyanide cleavage of unsymmetrical disulfides, J. Am. Chem. Soc., 83, 4644-4647.

47. Gawron, O. and Fernando, J. (1961) Kinetics of the cyanide-cystine reaction, J. Am. Chem. Soc., 83, 2906-2908.

48. Schneider, J. F. and Westley, J. (1969) Metabolic interrelations of sulfur in proteins, thiosulfate and cystine, J. Biol. Chem., 244, 5735-5744.

49. Gawron, O., Mahboob, S. and Fernando, J. (1964) pH Effects on the kinetics of the cystine-cyanide reaction, J. Am. Chem. Soc., 86, 2283-2286.

50. Kawamura, S., Otsuji, Y., Nakabayashi, T., Kitao, T. and Tsurugi, J. (1965) Aralkyl hydrodisulfides. IV. The reaction of benzyl hydrodisulfide with several nucleophiles, J. Org. Chem., 30, 2711-2714.

51. Tsurugi, J., Nakabayashi, T. and Ishihara, T. (1965) Aralkyl hydrodisulfides. III. The reaction with tertiary phosphines, J. Org. Chem., 30, 2707-2710.

52. Kawamura, S., Kitao, T., Nakabayashi, T., Horii, T. and Tsurugi, J. (1968) Aralkyl hydrodisulfides. VIII. Alaline decomposition and its competition with nucleophiles, J. Org. Chem., 33, 1179-1181.

53. Bailey, T. S., Zakharov, L. N. and Pluth, M. D. (2015) Understanding hydrogen sulfide storage: Probing conditions for sulfide release from hydrodisulfides, J. Am. Chem. Soc., 136, 10573-10576.

54. Parker, A. J. and Kharasch, N. (1960) Derivatives of sulfenic acids. XXXVI. The ionic scission of the sulfur-sulfur bond. Part 1, J. Am. Chem. Soc., 82, 3071-3075.

55. Everett, S. A. and Wardman P. (1995) Perthiols as Antioxidants: Radical-scavenging and prooxidative mechanisms, Methods Enzymol., 251, 55-69. 
56. Kharasch, M. S., Nudenberg, W. and Mantell, G. J. (1951) Reactions of atoms and free radicals in solution. XXV. The reactions of olefins with mercaptans in the presence of oxygen, $J$. Org. Chem., 16, 524-532.

57. Misra, H. P. (1974) Generation of superoxide free radical during the autoxidation of thiols, J. Biol. Chem., 249, 2151-2155.

58. Bagiyan, G. A., Koroleva, I. K., Soroka, N. V. and Ufimtzev, A. V. (2004) Kinetics of the catalytic oxidation reactions of thiol compounds in aqueous solution in the presence of copper ions, Kinet. Catal., 45, 372-380.

59. Golchoubian, H. and Hosseinpoor, F. (2007) Aerobic oxidation of thiols to disulfides catalyzed by manganese(III) Schiff-base complex, Catalysis Comm., 8, 697-700.

60. Benson, S. W. (1978) Thermochemistry and kinetics of sulfur-containing molecules and radicals, Chem. Rev., 78, 23-35.

61. Denu, J. M. and Tanner, K. G. (1998) Specific and reversible inactivation of protein tyrosine phosphatases by hydrogen peroxide: Evidence for a sulfenic acid intermediate and implications for redox regulation, Biochemistry, 37, 5633-5642.

62. Salmeen, A., Andersen, J. N., Myers, M. P., Meng, T.-C., Hinks, J. A., Tonks, N. K. and Barford, D. (2003) Redox regulation of protein tyrosine phosphatase $1 \mathrm{~B}$ involves a sulphenylamide intermediate, Nature, 423, 769-773.

63. van Montfort, R. L. M., Congreve, M., Tisi, D., Carr, R. and Jhoti, H. (2003) Oxidation state of the active-site cysteine in protein tyrosine phosphatase 1B, Nature, 423, 773-777.

64. Sivaramakrishnan, S., Cummings, A. H. and Gates, K. S. (2010) Protection of a singlecysteine redox switch from oxidative destruction: On the functional role of sulfenyl amide formation in the redox-regulated enzyme PTP1B, Bioorg. Med. Chem. Lett., 20, 444-447.

65. Bunnett, J. F. (1963) Nucleophilic reactivity, Annu. Rev. Phys. Chem., 14, 271-290. 
66. Jaramillo, P., Perez, P., Contreras, R., Tiznado, W. and Fuentealba, P. (2006) Definition of a nucleophilicty scale, J. Phys. Chem., 110, 8181-8187. 